\title{
Total Synthesis of Gobiusxanthin Stereoisomers and Their Application to Determination of Absolute Configurations of Natural Products: Revision of Reported Absolute Configuration of Epigobiusxanthin
}

\section{Yumiko Yamano ${ }^{1, *}$, Kotaro Ematsu ${ }^{1}$, Hiromasa Kurimoto ${ }^{1}$, Takashi Maoka ${ }^{2}$ and Akimori Wada ${ }^{1}$}

1 Department of Organic Chemistry for Life Science, Kobe Pharmaceutical University, Motoyamakita-machi, Higashinada-ku, Kobe 658-8558, Japan;

E-Mails: hm090930@st.kobepharma-u.ac.jp (H.K.); a-wada@kobepharma-u.ac.jp (A.W.)

2 Research Institute for Production Development, 15 Shimogamo-morimoto-cho, Sakyo-ku, Kyoto 606-0805, Japan; E-Mail: maoka@ mbox.kyoto-inet.or.jp

* Author to whom correspondence should be addressed; E-Mail: y-yamano@kobepharma-u.ac.jp; Tel./Fax: +81-78-441-7562.

Academic Editor: Orazio Taglialatela-Scafati

Received: 4 December 2014 / Accepted: 22 December 2014 / Published: 30 December 2014

\begin{abstract}
R)-Gobiusxanthin stereoisomers (1a-d) were synthesized by stereoselective Wittig reaction of the ( $3 R)$ - $\mathrm{C}_{15}$-acetylenic tri- $n$-butylphosphonium salt 7 with $\mathrm{C}_{25}$-apocarotenal stereoisomers 5a,b and 14a,b bearing four kinds of 3,6-dihydroxy-e-end groups. The validity of the reported stereochemistry of gobiusxanthin was demonstrated by the fact that the reported spectral data of natural gobiusxanthin were in agreement with those of synthetic $\left(3 R, 3^{\prime} S, 6^{\prime} R\right)$-gobiusxanthin (1a). On the other hand, the reported CD spectral data of natural epigobiusxanthin, which has been assigned as $\left(3 R, 3^{\prime} R, 6^{\prime} R\right)$-isomer (3'-epigobiusxanthin), were identical with those of synthetic $\left(3 R, 3^{\prime} S, 6^{\prime} S\right)$-isomer $\mathbf{1 d}$ (6'-epigobiusxanthin) rather than those of the corresponding synthetic 3'-epi-isomer $\mathbf{1 b}$. It was found that the stereochemistry at C3-position has little effect on the shape of their $\mathrm{CD}$ spectra. Thus, in order to reinforce the validity of the absolute configurations at C3-position of natural specimens, $\left(3 S, 3^{\prime} S, 6^{\prime} R\right)$ - and $\left(3 S, 3^{\prime} S, 6^{\prime} S\right)$-stereoisomers $1 \mathbf{1 e}$ and $\mathbf{1 f}$ were also synthesized and a HPLC analytical method for four stereoisomers was established by using a column carrying a chiral stationary phase. The HPLC analysis has proven that the stereochemistry of the natural epigobiusxanthin is $3 R, 3^{\prime} S, 6 ' S$.
\end{abstract}


Keywords: carotenoid; gobiusxanthin; epigobiusxanthin; total synthesis; chiral HPLC separation; absolute configuration

\section{Introduction}

Gobiusxanthin (1a) (Figure 1), which bears a novel 3',6'-dihydroxy- $\varepsilon$-end group, was first isolated from the common freshwater goby Rhinogobius brunneus [1] and then from the salmon Oncorhynchus keta [2]. Its structure was determined to be 7,8-didehydro- $\beta, \varepsilon$-carotene-3,3',6'-triol by MS and ${ }^{1} \mathrm{H}-\mathrm{NMR}$ spectroscopies and the absolute configuration was tentatively assigned as $3 R, 3{ }^{\prime} S, 6^{\prime} R$ from the resemblance of its $C D$ spectrum to the calculated one of half ( $3 S, 6 S, 3 ' S, 6$ 'S)-tunaxanthin and half $\left(3 R, 3^{\prime} R\right)$-alloxanthin according to the additivity rule of $\mathrm{CD}$ spectra [3]. From the salmon Oncorhynchus keta, salmoxanthin and deepoxysalmoxanthin, possessing the same 3',6'-dihydroxy-e-end group, were also isolated together with gobiusxanthin [2]. Their absolute configurations were similarly postulated by comparing their CD spectra with those of analogous compounds. Recently, we accomplished the first total synthesis of these two carotenoids and consequently confirmed that their proposed configurations are correct [4]. The stereoisomer of gobiusxanthin, 3'-epigobiusxanthin (1b) was isolated from the crown-of-thorns starfish Acanthaster planci [5]. Its trans-configuration of the two hydroxy groups at C3' and C6' was determined by NOESY experiment and a $6 ' R$ configuration was estimated from the fact that it showed the negative Cotton effect around $280 \mathrm{~nm}$ in the CD spectrum [3]. In order to obtain an additional proof on the stereochemistries of gobiusxanthin (1a) and 3'-epigobiusxanthin (1) $)$, we expected that efficient combination of a sterically-defined synthesis of authentic stereoisomers, spectroscopic analyses including NMR and CD, and a HPLC separation using a chiral column could be beneficial.
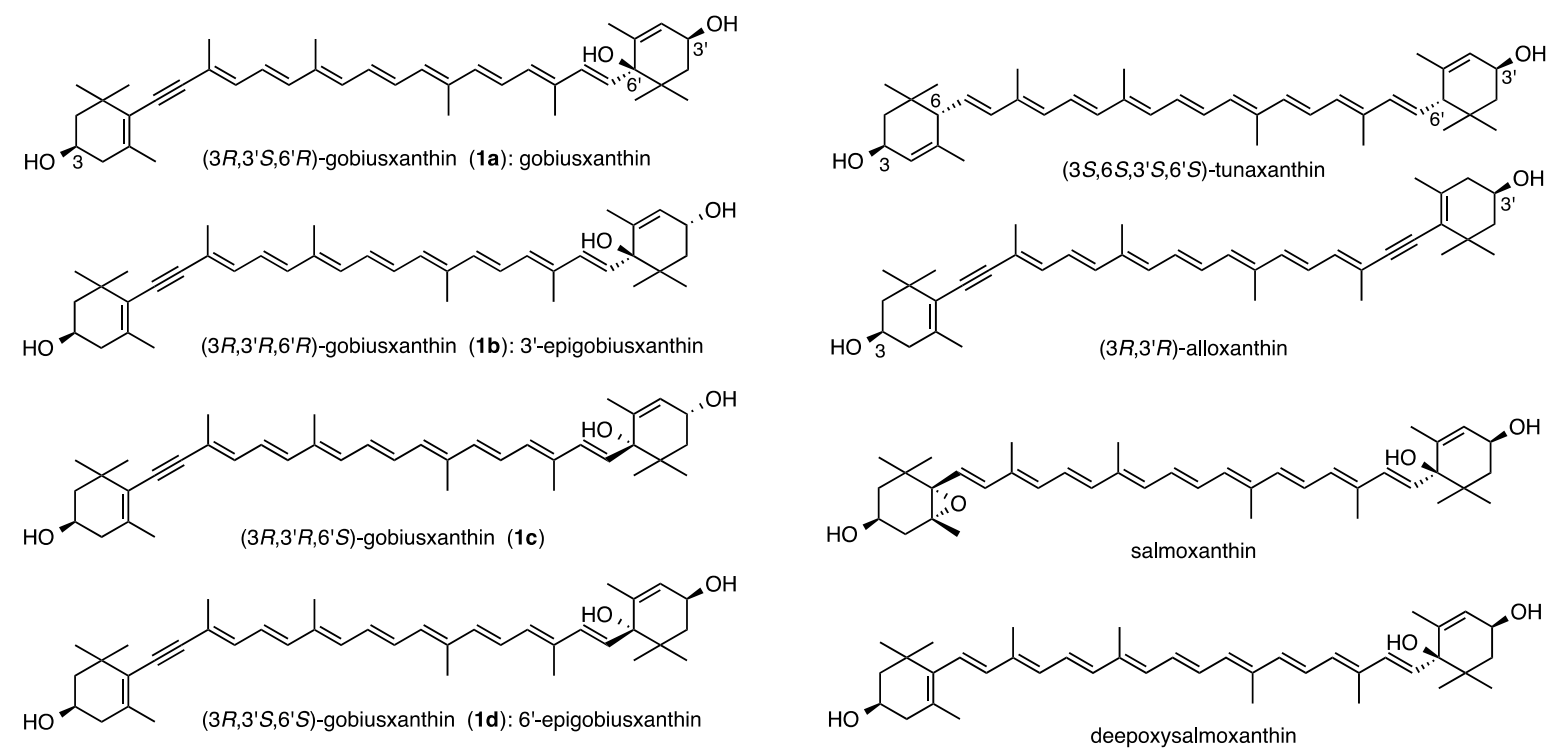

Figure 1. Cont. 

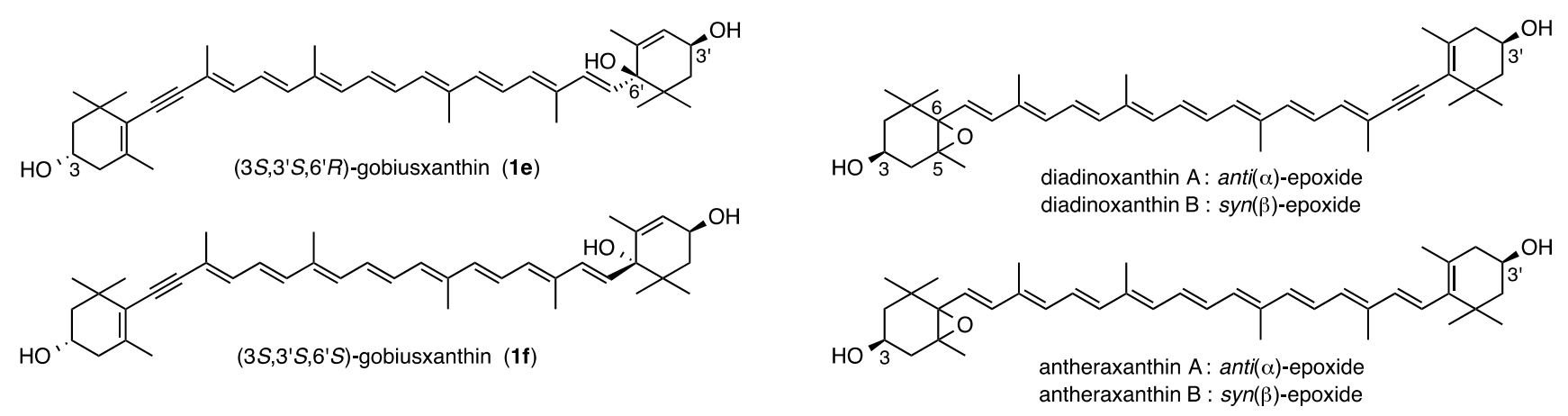

Figure 1. Structures of stereoisomers of gobiusxanthin $(\mathbf{1 a}-\mathbf{f})$ and other related carotenoids.

\section{Results and Discussion}

\subsection{Synthesis of Gobiusxanthin (1a) and 3'-Epigobiusxanthin (1b)}

We previously reported [6] that the $\mathrm{C}_{15}$-acetylenic tri- $n$-butylphophonium salt 7 (Scheme 1 ) is a useful tool for stereoselective synthesis of acetylenic carotenoids. In addition, the triethylsilyl (TES)-protected 3,6-syn-dihydroxydienoate $\mathbf{2 a}$ and the 3,6-anti-dihydroxydienoate $\mathbf{8}$ have already been prepared [4] in the course of synthesis of salmoxanthin. Thus, we synthesized gobiusxanthin (1a) and 3'-epigobiusxanthin (1b) by stereoselective Wittig reaction of the $\mathrm{C}_{15}$-phosphonium salt $\mathbf{7}$ with $\mathrm{C}_{25}$-apocarotenals $\mathbf{5 a}$ and $\mathbf{5 b}$, which were derived from compounds $\mathbf{2} \mathbf{a}$ and $\mathbf{8}$, as shown in Scheme 1.
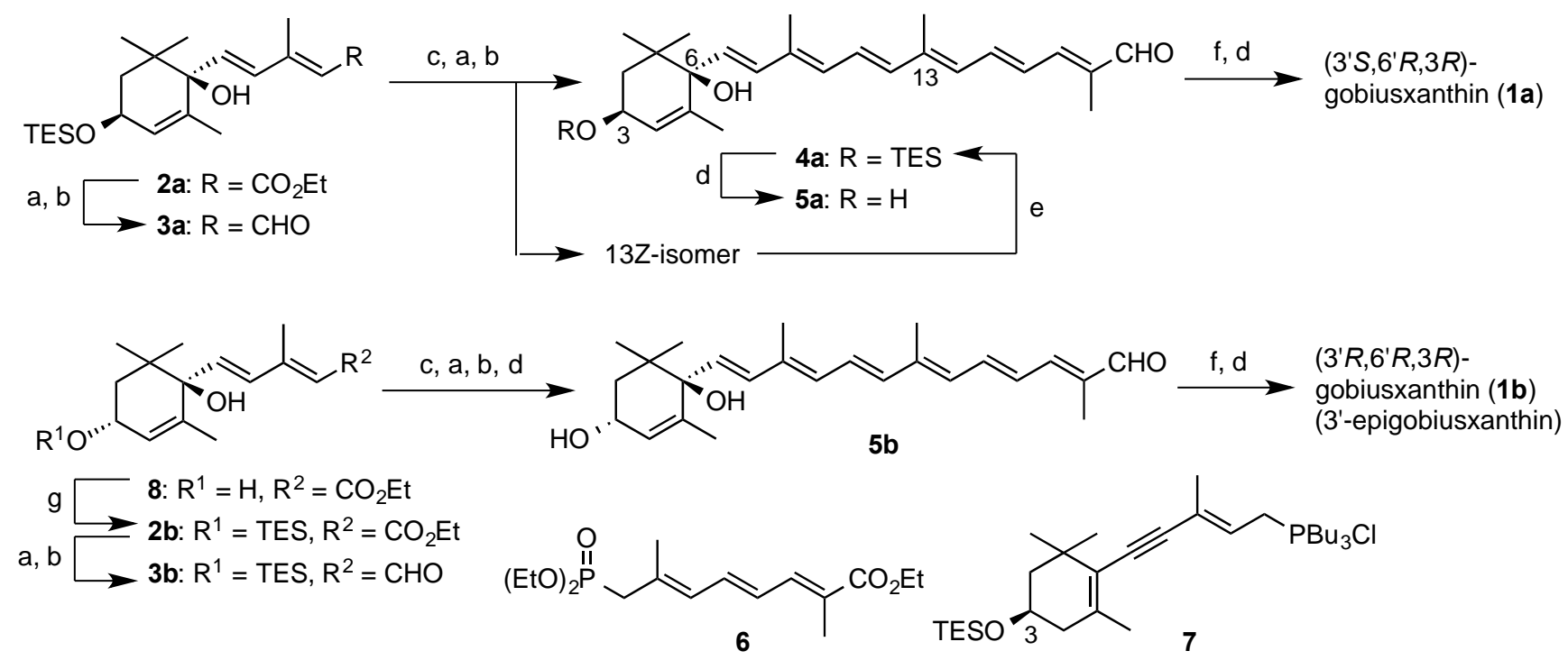

Scheme 1. Synthesis of gobiusxanthin (1a) and 3'-epigobiusxanthin (1b). Reagents:

(a) $\mathrm{LAH}$; (b) $\mathrm{MnO}_{2}$; (c) phosphonate 6, $n$-BuLi, $N, N^{\prime}$-dimethylpropyleneurea (DMPU);

(d) TBAF-AcOH; (e) $\mathrm{PdCl}_{2}\left(\mathrm{MeCN}_{2}, \mathrm{Et}_{3} \mathrm{~N}\right.$; (f) phosphonium salt 7, NaOMe; (g) TESCl.

The $\mathrm{C}_{15}$-syn-dihydroxydienoate 2a [4] was subjected to lithium aluminum hydride (LAH) reduction and subsequent $\mathrm{MnO}_{2}$ oxidation to afford the dienal 3a in $61 \%$ yield. This was then condensed with the previously reported $\mathrm{C}_{10}$-phosphonate 6 [7], and the resulting hexaenoate was reduced with $\mathrm{LAH}$ and followed by $\mathrm{MnO}_{2}$ oxidation to provide TES-protected all- $E$-apocarotenal 4a and its $13 Z$-isomer in $46 \%$ and $16 \%$ yield from 3a, respectively. The 13Z-isomer was converted (52\%) into the desired 
all- $E$-isomer by isomerization using a palladium catalyst $[4,8]$. After treatment of compound $\mathbf{4 a}$ with tetrabutylammonium fluoride (TBAF), the resulting deprotected apocarotenal $\mathbf{5 b}$ was condensed with phosphonium salt 7 under previously reported [6] conditions $\left(\mathrm{NaOMe}\right.$ in $\left.\mathrm{CH}_{2} \mathrm{Cl}_{2}\right)$ and then desilytated to provide gobiusxanthin in $69 \%$ over the 3 steps.

After protection (quant.) of $\mathrm{C}_{15}$-anti-dihydroxydienoate 8 [4], the resulting TES-ether $\mathbf{2 b}$ was transformed into 3'-epigobiusxanthin (1b) via the stereoselective condensation of $\mathrm{C}_{25}$-apocarotenal $\mathbf{5 b}$ with the $\mathrm{C}_{15}$-acetylenic tri- $n$-butylphophonium salt $\mathbf{7}$, in the same procedure as synthesis of gobiusxanthin (1a).

${ }^{1} \mathrm{H}-\mathrm{NMR}$ spectral data of synthetic $\mathbf{1 a}$ and ${ }^{1} \mathrm{H}$ - and ${ }^{13} \mathrm{C}-\mathrm{NMR}$ spectral data of synthetic $\mathbf{1 b}$ were in good agreement with those of the reported data of goboiusxanthin [1] and 3'-epigobiusxanthin [5], respectively. As shown in Figure 2, the CD spectrum of synthetic 1a was basically identical with the reported spectrum of gobiusxanthin, except for the intensities. On the other hand, the CD spectrum of the synthetic 1b was opposite in sign to that of the reported 3'-epigobiusxanthin, indicating the reported absolute stereochemistry needs a correction. To make sure the stereochemistry of natural epigobiusxanthin, (6'S)-stereoisomer was also prepared as a reference sample.

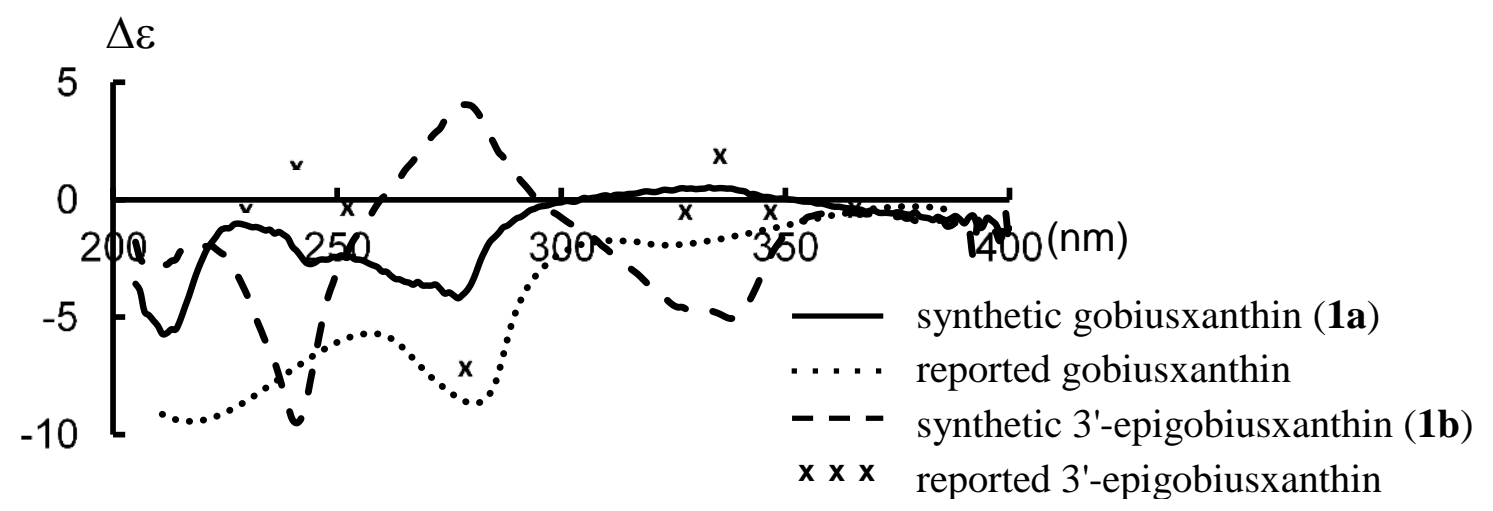

Figure 2. $\mathrm{CD}$ spectra in $\mathrm{Et}_{2} \mathrm{O}$-isopentane-EtOH (5:5:2) of synthetic gobiusxanthin (1a), 3 '-epigobiusxanthin (1b) and their reported spectra.

\subsection{Synthesis of Gobiusxanthin Stereoisomers $\mathbf{1 c}-\mathbf{1 f}$}

According to the procedure for the preparation of the corresponding enantiomer 8 [4], (3S,6S)-anti-diol 11b was prepared as shown in Scheme 2. Previously prepared anti $(\alpha)$-epoxide 9 [9] was oxidized with Dess-Martin periodinane (DMP) and then treated with a large amount of silica gel in AcOEt to afford (6S)-hydroxyenone $\mathbf{1 0}$ in $82 \%$ yield. Reduction of $\mathbf{1 0}$ with 9-borabicyclo[3.3.1]nonane (9-BBN) [4,10] gave (3S,6S)-anti-diol 11b (44\%), together with (3R,6S)-syn-diol 11a (47\%). Anti-diol 11b was converted into $(3 R, 3 ' S, 6 ' S)$-gobiusxanthin (1d) in the same procedure as synthesis of $\mathbf{1 a}$ and $\mathbf{1 b}$. $\left(3 R, 3^{\prime} R, 6^{\prime} S\right)$-Gobiusxanthin (1c) was also synthesized from syn-diol 11a.

As shown in Figure 3b, the reported CD spectral data [5] of natural epigobiusxanthin shown by " $x$ " were basically identical in the shape with those of synthetic $(3 R, 3 ' S, 6$ 'S)-isomer 1d (6'-epigobiusxanthin). It was found that the stereochemistry at C3-position has little effect on the shape of their CD spectra; CD spectrum of ( $\left.3 R, 3^{\prime} S, 6^{\prime} R\right)$-gobiusxanthin (1a) showed an antisymmetrical shape 
to that of $\left(3 R, 3^{\prime} R, 6^{\prime} S\right)$-gobiusxanthin (1c) (Figure $\left.3 \mathrm{a}\right)$, whereas that of $\left(3 R, 3^{\prime} R, 6^{\prime} R\right)$-gobiusxanthin $(\mathbf{1 b})$ showed an antisymmetrical shape to that of (3R,3'S,6'S)-gobiusxanthin (1d) (Figure 3b). The stereochemistry at C3'-position has a decisive influence on the shape and the sign of their CD spectra. It indicates that the $\mathrm{C} 3$-absolute configurations of gobiusxanthin and epigobiusxanthin cannot be determined exactly by CD spectra.

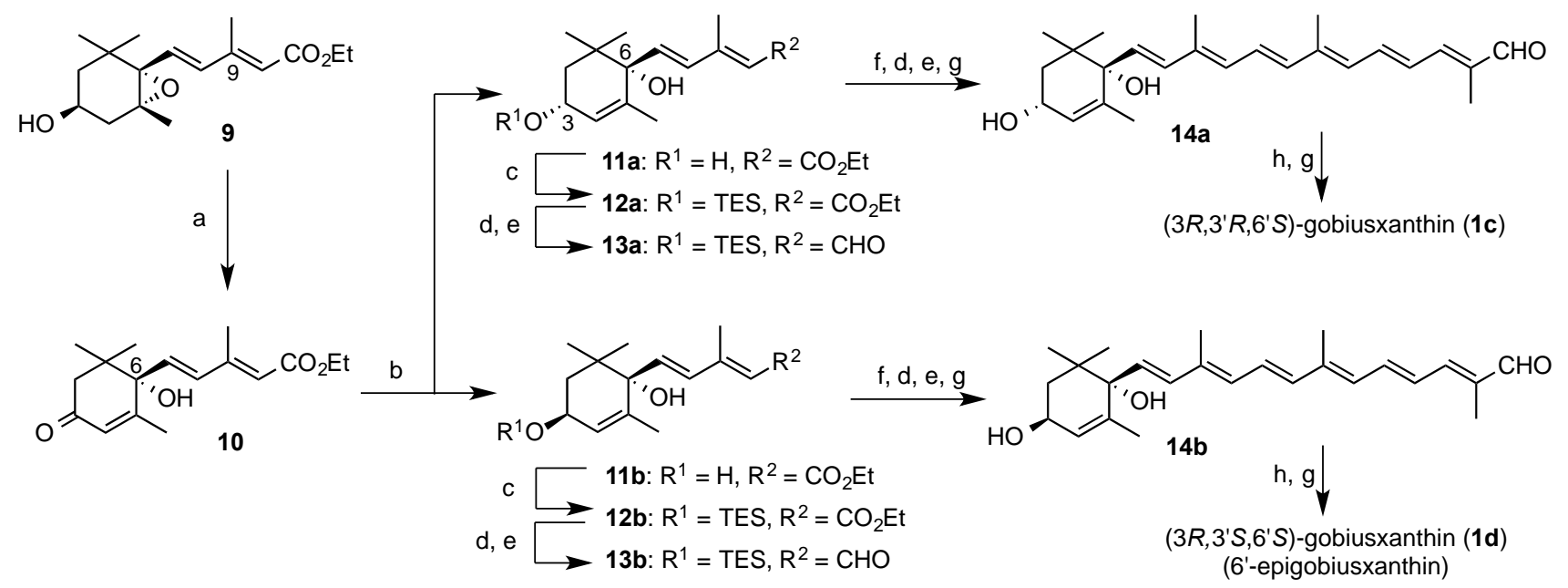

Scheme 2. Synthesis of (6'S)-gobiusxanthin stereoisomers 1c and 1d. Reagents: (a) DMP then $\mathrm{SiO}_{2}$; (b) 9-BBN; (c) TESCl; (d) $\mathrm{LAH}$; (e) $\mathrm{MnO}_{2}$; (f) phosphonate 6, $n$-BuLi, DMPU; (g) TBAF-AcOH; (h) phosphonium salt 7, NaOMe.

(a) Syn-dihydroxy compounds

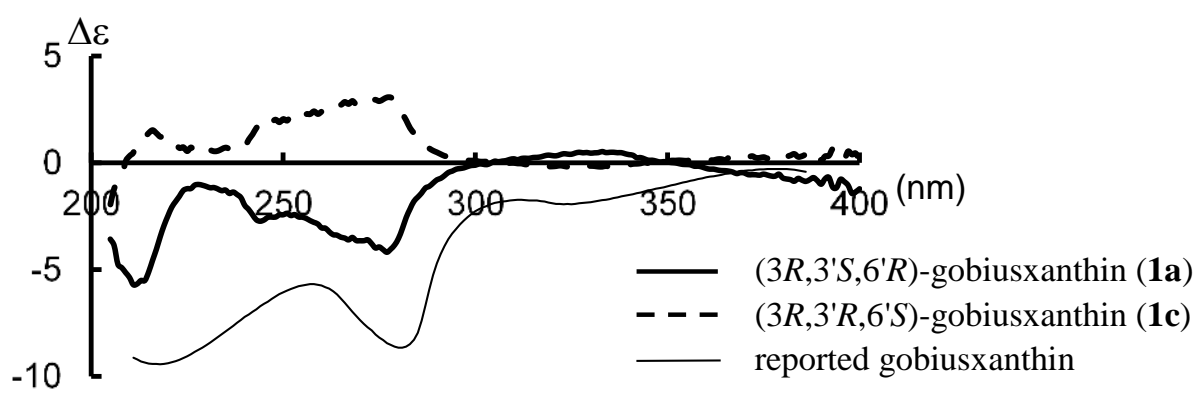

(b) Anti-dihydroxy compounds

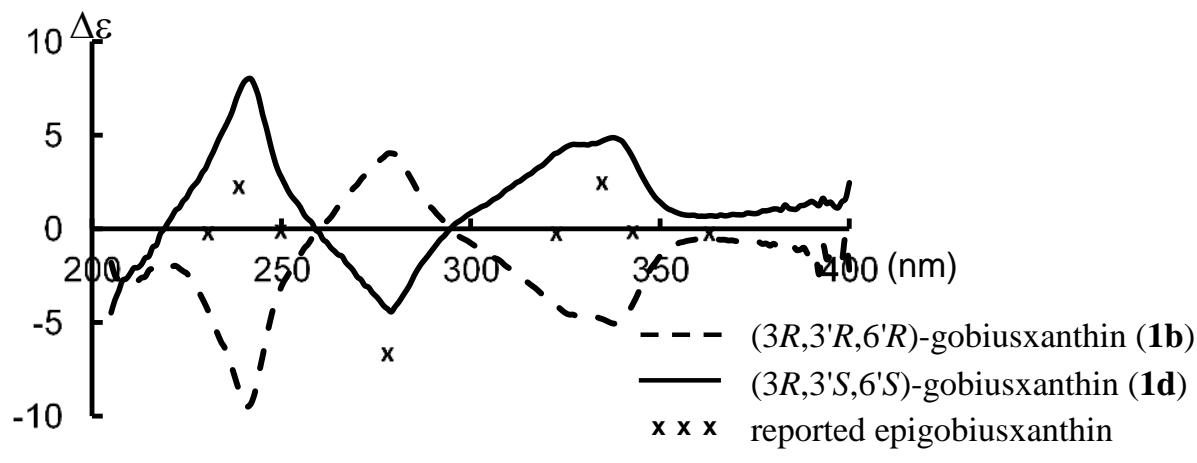

Figure 3. $\mathrm{CD}$ spectra in $\mathrm{Et}_{2} \mathrm{O}$-isopentane-EtOH $(5: 5: 2)$ of $(3 R)$-gobiusxanthin stereoisomers (1a-d) and reported spectra of gobiusxanthin and 3'-epigobiusxanthin. 
Previously, we reported [11] that most alloxanthin specimens (Figure 1) isolated from various aquatic animals consist of only $\left(3 R, 3^{\prime} R\right)$-stereoisomer; however, those from some aquatic animals are exceptionally mixtures of three stereoisomers. Thus, in order to reinforce the validity of the absolute configurations at C3-position of natural specimens, (3S,3'S,6'R)- and (3S,3'S,6'S)-stereoisomers 1e and 1f were also synthesized by Wittig condensation of $\mathrm{C}_{25}$-apocarotenals $\mathbf{5 a}$ and $\mathbf{1 4 b}$ with the enantiomers [11] of $\mathrm{C}_{15}$-phosphonium salt $\mathbf{7}$ and a HPLC analytical method for four stereoisomers 1a, 1d, 1e and 1f was investigated. As a result, these stereoisomers can be separated using a chiral column (CHIRALPAK IB; Daicel, Tokyo, Japan) as shown in Figure 4.

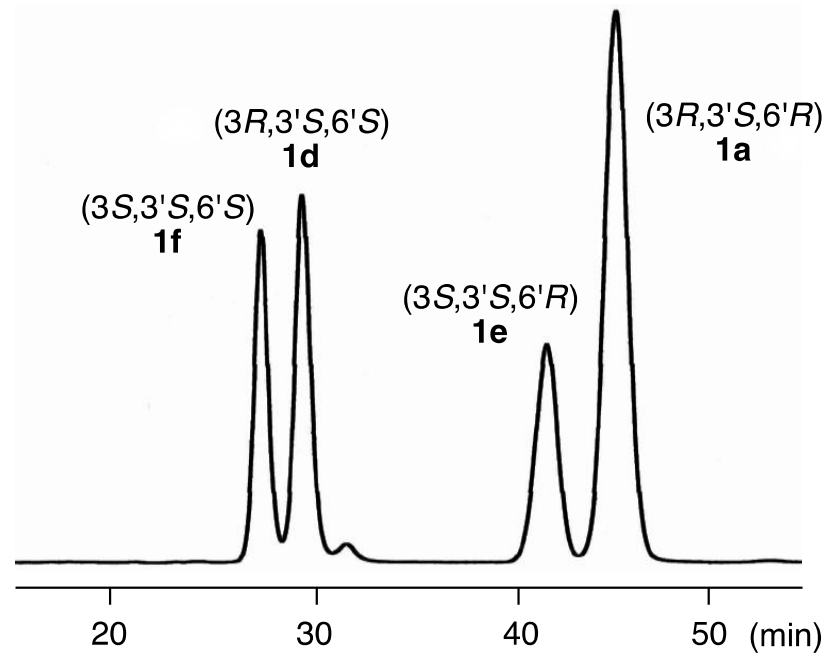

Figure 4. HPLC elution profile of a mixture of four stereoisomers of gobiusxanthin. Column: CHIRALPAK IB $0.46 \times 25 \mathrm{~cm}$; eluent: $\mathrm{EtOH}-n$-hexane- $\mathrm{CH}_{2} \mathrm{Cl}_{2}$ (1:75:25); flow rate: $1.0 \mathrm{~mL} / \mathrm{min}$; temperature: $23{ }^{\circ} \mathrm{C}$; detection: $450 \mathrm{~nm}$.

Application of this method to epigobiusxanthin isolated from the crown-of-thorns starfish Acanthaster planci [5] demonstrated that its stereochemistry is $3 R, 3 ' S, 6$ 'S. As described above, the absolute stereochemistries at C3'- and C6'-positions of gobiusxanthin were successfully to be 3'S and $6 ' R$ based of their identical CD spectra. Once we get gobiusxanthin specimen from a natural source, the absolute stereochemistry of C3-position can be unambiguously determined by the HPLC analysis.

The syn-configuration of the hydroxyl group at $\mathrm{C} 3$ and the epoxide oxygen was firstly identified in carotenoids, diadinoxanthin B and antheraxanthin B (Figure 1), isolated from the common freshwater goby Rhinogobius brunneus [1]. Diadinoxanthin B would be involved in a biosynthetic pathway of gobiusxanthin. On the contrary, epigobiusxanthin, which was found to be 6'-epi-form rather than 3'-epi-form, would be derived from common anti( $\alpha$-epoxy carotenoids such as diadinoxanthin A.

\section{Experimental Section}

\subsection{General}

UV-VIS spectra were recorded on a JASCO V-650 instrument (JASCO, Tokyo, Japan), with ethanol solutions. IR spectra were measured on a Perkin-Elmer spectrum 100 FT-IR spectrometer (Perkin-Elmer, Yokohama, Japan), with chloroform solutions. ${ }^{1} \mathrm{H}-$ and ${ }^{13} \mathrm{C}-\mathrm{NMR}$ spectra were 
determined on a Varian Gemini-300 or a VXR-500 superconducting FT-NMR spectrometer (Agilent Technologies, Santa Clara, CA, USA), with deuteriochloroform solutions (tetramethylsilane as the internal reference). $J$ Values are given in Hz. Mass spectra were taken on a Thermo Fisher Scientific Exactive spectrometer (Thermo Fisher Scientific, Bremen, Germany). CD spectra were measured on a Shimadzu-AVIN 62A DS circular dichroism spectrometer (Shimadzu, Kyoto, Japan). The concentrations were calculated using $\log \varepsilon=5.0$ at main $\lambda_{\max }$ (in EPA). Optical rotations were measured on a JASCO P-2200 polarimeter (JASCO, Tokyo, Japan).

HPLC analyses were performed on Simadzu-LC-20AT instrument (Shimadzu, Kyoto, Japan) with a photodiode array detector (Waters 996, Tokyo, Japan) and column oven (GL Sciences Model 552, Tokyo, Japan). Flash column chromatography (CC) was performed on using Kanto Silica Gel 60 N. Preparative HPLC was carried out on a Shimadzu LC-6A with a UV-VIS detector (Shimadzu, Kyoto, Japan).

All operations were carried out under nitrogen or argon. Evaporation of the extract or the filtrate was carried out under reduced pressure. Ether refers to diethyl ether, and hexane to $n$-hexane. NMR assignments are given using the carotenoid numbering system.

\subsection{Synthesis of Gobiusxanthin (1a) and 3'-Epigobiusxanthin (1b)}

(2E,4E)-5-[(1R,4S)-1-Hydroxy-2,6,6-trimethyl-4-triethylsilyloxycyclohex-2-en-1-yl]-3-methylpenta-2,4-dienal (3a). A solution of ester 2a [4] (1.27 g, $3.11 \mathrm{mmol})$ in dry ether (15 mL) was added dropwise to a stirred suspension of LAH $(118 \mathrm{mg}, 3.11 \mathrm{mmol})$ in dry ether $(20 \mathrm{~mL})$ at $0{ }^{\circ} \mathrm{C}$. After being stirred at $0{ }^{\circ} \mathrm{C}$ for $10 \mathrm{~min}$, the excess of LAH was decomposed by dropwise addition of moist ether and the mixture was filtered through a pad of Celite. The filtrate was dried and evaporated to give the crude alcohol, which without purification, was dissolved in ether $(15 \mathrm{~mL})$ and hexane $(15 \mathrm{~mL})$ and stirred with $\mathrm{MnO}_{2}(7.5 \mathrm{~g})$ at $\mathrm{rt}$ for $30 \mathrm{~min}$. After $\mathrm{MnO}_{2}$ was filtered off, the filtrate was concentrated. The resulting residue was purified by flash CC (AcOEt-hexane, 3:7) to afford the aldehyde 3a (697 mg, 61\% from 2a): [ $\alpha]_{D^{22}}-149.1$ ( $c$ 1.26, MeOH); IR v 3600, 3447 (OH), 1661 (conj. CO), 1631, $1600(\mathrm{C}=\mathrm{C}) ;{ }^{1} \mathrm{H}-\mathrm{NMR}(300 \mathrm{MHz}) \delta 0.63(6 \mathrm{H}, \mathrm{q}, J$ 8, SiCH $\times 3), 0.95,0.97$ (each 3H, s, gem-Me), $0.98\left(9 \mathrm{H}, \mathrm{t}, J\right.$ 8, $\left.\mathrm{CH}_{2} \mathrm{Me} \times 3\right), 1.64(3 \mathrm{H}, \mathrm{t}, J 1.5,5-\mathrm{Me}), 1.68\left(2 \mathrm{H}, \mathrm{m}, 2-\mathrm{H}_{2}\right), 2.27(3 \mathrm{H}, \mathrm{d}, J 1,9-\mathrm{Me})$, $4.26(1 \mathrm{H}, \mathrm{m}, 3-\mathrm{H}), 5.56(1 \mathrm{H}, \mathrm{br} \mathrm{s}, 4-\mathrm{H}), 6.01(1 \mathrm{H}, \mathrm{br} \mathrm{d}, J 8,10-\mathrm{H}), 6.17(1 \mathrm{H}, \mathrm{d}, J 16,7-\mathrm{H}), 6.56(1 \mathrm{H}, \mathrm{d}$, $J$ 16, 8-H), $10.12(1 \mathrm{H}, \mathrm{d}, J 8, \mathrm{CHO}) ;{ }^{13} \mathrm{C}-\mathrm{NMR}(75 \mathrm{MHz}) \delta 4.79(\mathrm{C} \times 3), 6.80(\mathrm{C} \times 3), 13.32,19.26$, 24.38, 24.46, 38.52, 42.60, 65.29, 77.39, 129.18, 129.43, 133.29, 136.77, 138.82, 153.68, 191.49; HRMS (ESI) $m / z$ calcd for $\mathrm{C}_{21} \mathrm{H}_{36} \mathrm{O}_{3} \mathrm{NaSi}(\mathrm{M}+\mathrm{Na})^{+}$387.2326, found 387.2317.

(2E,4E,6E,8E,10E,12E)-13-[(1R,4S)-1-Hydroxy-2,6,6-trimethyl-4-triethylsilyloxycyclohex-2-en-1yl]-2,7,11-trimethyltrideca-2,4,6,8,10,12-hexaenal (4a). To a solution of C10-phosphonate 6 [7] $(1.90 \mathrm{~g}, 5.76 \mathrm{mmol})$ and $N, N^{\prime}$-dimethylpropyleneurea (DMPU) $(0.69 \mathrm{~mL}, 5.76 \mathrm{mmol})$ in dry THF $(20 \mathrm{~mL})$ was added $n$-BuLi $(1.63 \mathrm{M}$ in hexane; $3.64 \mathrm{~mL}, 5.94 \mathrm{mmol})$ at $-20{ }^{\circ} \mathrm{C}$. After being stirred at $-20{ }^{\circ} \mathrm{C}$ for $15 \mathrm{~min}$, a solution of the aldehyde $3 \mathbf{a}(700 \mathrm{mg}, 1.92 \mathrm{mmol})$ in dry THF (10 mL) was added to the reaction mixture and stirring was continued at $-20{ }^{\circ} \mathrm{C}$ for $20 \mathrm{~min}$. After being quenched with saturated aq. $\mathrm{NH}_{4} \mathrm{Cl}$, the mixture was extracted with AcOEt. The extracts were washed with brine, dried and evaporated to give a residue, which was purified by flash CC (ether-hexane, 3:7) to provide an isomeric mixture of the hexaenoate (786 mg, 76\%) as an yellow oil: IR $v 3599,3518(\mathrm{OH}), 1692$ (conj. 
$\mathrm{CO}$ ), 1614, 1602, $1555(\mathrm{C}=\mathrm{C})$; HRMS (ESI) $\mathrm{m} / \mathrm{z}$ calcd for $\mathrm{C}_{33} \mathrm{H}_{52} \mathrm{O}_{4} \mathrm{NaSi}(\mathrm{M}+\mathrm{Na})^{+}$563.3527, found 353.3516 .

A solution of this isomeric mixture in dry ether $(20 \mathrm{~mL})$ was added dropwise to a stirred suspension of LAH ( $83 \mathrm{mg}, 2.2 \mathrm{mmol})$ in dry ether $(20 \mathrm{~mL})$ at $0{ }^{\circ} \mathrm{C}$. After being stirred at $0{ }^{\circ} \mathrm{C}$ for $15 \mathrm{~min}$, the excess of LAH was decomposed by dropwise addition of moist ether and the mixture was filtered through a pad of Celite. The filtrate was dried and evaporated to give the crude diol, which without purification, was dissolved in THF $(1 \mathrm{~mL})$, ether $(15 \mathrm{~mL})$ and hexane $(15 \mathrm{~mL})$, and then stirred with $\mathrm{MnO}_{2}(5.3 \mathrm{~g})$ at room temperature (rt) for $30 \mathrm{~min}$. After $\mathrm{MnO}_{2}$ was filtered off, the filtrate was concentrated. The resulting residue was purified by flash CC (AcOEt-hexane, 3:7) and then preparative HPLC [LiChrosorb Si $60(7 \mu \mathrm{m}) 2 \times 25 \mathrm{~cm}$; ether-hexane, 27:73] to afford all- $E$-apocarotenal $4 \mathbf{a}$ (434 mg, 46\% from 3a) and its 13Z-isomer (149 mg, 16\% from 3a), as an orange foam, respectively.

all-E-Isomer 4a: UV-VIS $\lambda$ 421; IR v 3598, $3521(\mathrm{OH}), 1661$ (conj. CO), 1611, 1601, 1550 (C=C); ${ }^{1} \mathrm{H}-\mathrm{NMR}(300 \mathrm{MHz}) \delta 0.63\left(6 \mathrm{H}, \mathrm{q}, J\right.$ 8, $\left.\mathrm{SiCH}_{2} \times 3\right), 0.94,0.98($ each $3 \mathrm{H}, \mathrm{s}$, gem-Me), $0.98(9 \mathrm{H}, \mathrm{t}, J 8$, $\left.\mathrm{CH}_{2} \mathrm{Me} \times 3\right), 1.65$ (3H, t, J 1.5, 5-Me), 1.68 (2H, m, 2- $\left.\mathrm{H}_{2}\right), 1.88,1.94,2.04$ (each 3H, s, 9-Me, 13-Me, 13'-Me), 4.25 (1H, m, 3-H), $5.53(1 \mathrm{H}, \mathrm{m}, 4-\mathrm{H}), 5.67(1 \mathrm{H}, \mathrm{d}, J 15.5,7-\mathrm{H}), 6.23(1 \mathrm{H}, \mathrm{br} \mathrm{d}, J 11.5,10-\mathrm{H})$, $6.31(1 \mathrm{H}$, br d, $J 12,14-\mathrm{H}), 6.38(1 \mathrm{H}, \mathrm{d}, J 15,12-\mathrm{H}), 6.45(1 \mathrm{H}, \mathrm{d}, J 15.5,8-\mathrm{H}), 6.68(1 \mathrm{H}, \mathrm{dd}, J 12$, 14.5, 15'-H), 6.77 (1H, dd, $J 11.5,15,11-\mathrm{H}), 6.96$ (1H, br d, $\left.J 12,14^{\prime}-\mathrm{H}\right), 7.03(1 \mathrm{H}, \mathrm{dd}, J 12,14.5,15-\mathrm{H})$, $9.45(1 \mathrm{H}, \mathrm{s}, \mathrm{CHO}) ;{ }^{13} \mathrm{C}-\mathrm{NMR}(75 \mathrm{MHz}) \delta 4.82(\mathrm{C} \times 3), 6.83(\mathrm{C} \times 3), 9.54,13.00,13.14,19.35,24.40$, 24.45, 38.39, 42.73, 65.44, 77.42, $127.40(\mathrm{C} \times 2), 128.49,130.70,131.03,131.20,134.45,136.50$, $136.85(\mathrm{C} \times 2), 137.62,137.82,141.53,148.85,194.41$; HRMS $(\mathrm{ESI}) \mathrm{m} / \mathrm{z}$ calcd for $\mathrm{C}_{31} \mathrm{H}_{48} \mathrm{O}_{3} \mathrm{NaSi}$ $(\mathrm{M}+\mathrm{Na})^{+} 519.3265$, found 519.3257 .

13Z-Isomer: UV-VIS $\lambda$ 295, 410; IR v 3598, 3521 (OH), 1660 (conj. CO), 1612, 1598, 1565 (C=C); ${ }^{1} \mathrm{H}-\mathrm{NMR}(300 \mathrm{MHz}) \delta 0.63\left(6 \mathrm{H}, \mathrm{q}, J\right.$ 8, $\left.\mathrm{SiCH}_{2} \times 3\right), 0.94,0.99($ each $3 \mathrm{H}, \mathrm{s}$, gem-Me) $0.99(9 \mathrm{H}, \mathrm{t}, J 8$,

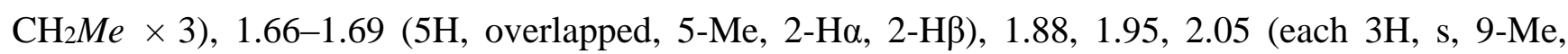
13-Me, 13'-Me), 4.26 (1H, m, 3-H), 5.53 (1H, s, 4-H), 5.69 (1H, d, J 16, 7-H), 6.16 (1H, d, J 12, 14-H), 6.27 (1H, d, $J 11,10-\mathrm{H}), 6.47$ (1H, dd, $J 16,8-\mathrm{H}), 6.62$ (1H, dd, $J 11.5,14,15-\mathrm{H}), 6.75$ (1H, dd, $J 11$, 15, 11-H), 6.90 (1H, d, J 15, 12-H), $6.97\left(1 \mathrm{H}, \mathrm{d}, J 11.5,14^{\prime}-\mathrm{H}\right), 7.03$ (1H, dd, $\left.J 12,14,15-\mathrm{H}\right), 9.45$ $(1 \mathrm{H}, \mathrm{s}, \mathrm{CHO}) ;{ }^{13} \mathrm{C}-\mathrm{NMR}(75 \mathrm{MHz}) \delta 4.82,(\mathrm{C} \times 3) 6.84(\mathrm{C} \times 3), 9.52,13.20,19.37,20.92,24.41$, 24.47, 38.42, 42.73, 65.45, 77.42, 126.60, 128.38, 128.55, 128.57, 129.36, 130.96, 131.09, 134.39, 136.36, 136.91, 137.10, 137.80, 140.22, 148.96, 194.49; HRMS (ESI) $\mathrm{m} / z$ calcd for $\mathrm{C}_{31} \mathrm{H}_{48} \mathrm{O}_{3} \mathrm{NaSi}$ $(\mathrm{M}+\mathrm{Na})^{+} 519.3265$, found 519.3255 .

Isomerization of $13 Z$-isomer of compound 4a. A solution $(2 \mathrm{~mL})$ prepared from $\mathrm{PdCl}_{2}\left(\mathrm{MeCN}_{2}\right.$ $(13 \mathrm{mg}), \mathrm{Et}_{3} \mathrm{~N}(7 \mathrm{~mL})$ and water $(1.2 \mathrm{~mL})$ in $\mathrm{MeCN}(8.8 \mathrm{~mL})$ was added to a solution of 13Z-isomer of compound $4 \mathbf{a}(103 \mathrm{mg})$ in $\mathrm{MeCN}(18 \mathrm{~mL})$ and the mixture was stirred at $\mathrm{rt}$ for $3 \mathrm{~h}$. The solvent was evaporated off to give a residue, which was purified by the same method described above to provide the all-E-isomer $\mathbf{4 a}(54 \mathrm{mg}, 52 \%)$.

(2E,4E,6E,8E,10E,12E)-13-[(1R,4S)-1,4-Dihydroxy-2,6,6-trimethylcyclohex-2-en-1-yl]-2,7,11trimethyltrideca-2,4,6,8,10,12-hexaenal (5a). To a stirred solution of TES ether 4a (434 mg, $0.86 \mathrm{mmol})$ in dry THF $(9 \mathrm{~mL})$ was added AcOH $(1 \mathrm{M}$ in THF; $0.30 \mathrm{~mL}, 0.30 \mathrm{mmol})$ and then TBAF (1 M in THF; $1.31 \mathrm{~mL}, 1.31 \mathrm{mmol}$ ) at $\mathrm{rt}$. After being stirred at $\mathrm{rt}$ for $5 \mathrm{~min}$, the mixture was evaporated 
to afford a residue, which was purified by flash CC (acetone-hexane, 35:65) to afford compound 5a (280 mg, 84\%) as orange solids: UV-VIS $\lambda$ 421; IR $v$ 3603, 3446 (OH), 1660 (conj. CO), 1611, 1601, $1550(\mathrm{C}=\mathrm{C}) ;{ }^{1} \mathrm{H}-\mathrm{NMR}(500 \mathrm{MHz}) \delta 0.94,1.02$ (each 3H, s, gem-Me), 1.53, 1.62 (each $1 \mathrm{H}$, br s, OH $\left.\times 2\right)$, 1.67 (1H, dd, J 7.5, 13.5, 2-H), 1.68 (3H, t, J 2, 5-Me), 1.81 (1H, dd, J 6.5, 13.5, 2-H), 1.89 (3H, s, 13'-Me), $1.94(3 \mathrm{H}, \mathrm{s}, 9-\mathrm{Me}), 2.04(3 \mathrm{H}, \mathrm{s}, 13-\mathrm{Me}), 4.25(1 \mathrm{H}, \mathrm{m}, 3-\mathrm{H}), 5.64(1 \mathrm{H}$, br s, 4-H), $5.69(1 \mathrm{H}, \mathrm{d}, J$ 15.5, 7-H), 6.24 (1H, br d, J 11.5, 10-H), 6.31 (1H, br d, J 12, 14-H), 6.39 (1H, d, J 15, 12-H), $6.42(1 \mathrm{H}, \mathrm{d}$, $J 15.5,8-\mathrm{H}), 6.70\left(1 \mathrm{H}, \mathrm{dd}, J 11.5,14.5,15^{\prime}-\mathrm{H}\right), 6.76(1 \mathrm{H}, \mathrm{dd}, J 11.5,15,11-\mathrm{H}), 6.96(1 \mathrm{H}, \mathrm{br} \mathrm{d}, J 11.5$, 14'-H), 7.02 (1H, dd, $J$ 12, 14.5, 15-H), 9.45 (1H, s, CHO); ${ }^{13} \mathrm{C}-\mathrm{NMR}(125 \mathrm{MHz}) \delta 9.59$ (13'-Me), 13.03 (13-Me), 13.22 (9-Me), 19.21 (5-Me), 24.37 (1-Me), 24.40 (1-Me), 38.19 (C1), 42.44 (C2), 65.24 (C3), 77.83 (C6), 127.29 (C11), 127.35 (C4), 127.55 (C15'), 130.74 (C7), 131.19 (C14), 131.50 (C10), 134.44 (C8), 136.31 (C9), 136.99 (C13'), 137.10 (C12), 137.59 (C15), 138.85 (C5), 141.48 (C13), 148.83 (C14'), 194.47 (CHO); HRMS (ESI) $m / z$ calcd for $\mathrm{C}_{25} \mathrm{H}_{34} \mathrm{O}_{3} \mathrm{NaSi}(\mathrm{M}+\mathrm{Na})^{+} 405.2400$, found 405.2393 .

(3R,3'S,6'R)-Gobiusxanthin (1a). NaOMe (1 M in $\mathrm{MeOH}$; $0.45 \mathrm{~mL}, 0.45 \mathrm{mmol})$ was added to a solution of the phosphonium salt 7 [6] (192 $\mathrm{mg}, 0.34 \mathrm{mmol})$ and the apocarotenal $\mathbf{5 a}(86 \mathrm{mg}, 0.23 \mathrm{mmol})$ in $\mathrm{CH}_{2} \mathrm{Cl}_{2}(10 \mathrm{~mL})$ at $\mathrm{rt}$. After being stirred at $\mathrm{rt}$ for $10 \mathrm{~min}$, the mixture was poured into saturated aq. $\mathrm{NH}_{4} \mathrm{Cl}$ and extracted with AcOEt. The extracts were washed with brine, dried and evaporated to give a residue, which was purified by flash $\mathrm{CC}$ (acetone-hexane, 3:7) to provide the crude condensed products. This was dissolved in dry THF $(9 \mathrm{~mL})$ and then $\mathrm{AcOH}(1 \mathrm{M}$ in THF; $0.30 \mathrm{~mL}, 0.30 \mathrm{mmol})$ and TBAF (1 $\mathrm{M}$ in THF; $0.42 \mathrm{~mL}, 0.42 \mathrm{mmol}$ ) were added to it at $\mathrm{rt}$. After being stirred at $\mathrm{rt}$ for $30 \mathrm{~min}$, the mixture was concentrated. The resulting residue was purified by flash $\mathrm{CC}$ (AcOEt- $\left.\mathrm{CH}_{2} \mathrm{Cl}_{2}-\mathrm{MeOH}, 25: 72: 3\right)$ and then preparative HPLC [LiChrosorb Si 60 (7 $\left.\mu \mathrm{m}\right) 2 \times 25 \mathrm{~cm}$; AcOEt- $\left.\mathrm{CH}_{2} \mathrm{Cl}_{2}-\mathrm{MeOH}, 10: 40: 1\right]$ to give (3R,3'S,6'R)-gobiusxanthin (1a) (90 mg, 69\% from 5a) as orange solids. ${ }^{1} \mathrm{H}-\mathrm{NMR}$ spectral data of this synthetic 1a were identical with those reported [1]: UV-VIS $\lambda 277,424,447,477$; CD $\left(9.83 \times 10^{-5} \mathrm{~mol} / \mathrm{L}, \mathrm{EPA}\right) \lambda(\Delta \varepsilon) 211(-5.7), 228(-1.0), 244(-2.7)$, 277 (-4.2), $304(0), 333(+0.5), 351(0)$; IR v 3604, $3448(\mathrm{OH}), 2172(\mathrm{C} \equiv \mathrm{C}), 1568(\mathrm{C}=\mathrm{C}) ;{ }^{1} \mathrm{H}-\mathrm{NMR}$ (500 MHz); $\delta 0.94$ (3H, s, 1'-Me $\alpha), 1.02\left(3 \mathrm{H}, \mathrm{s}, 1^{\prime}-\mathrm{Me} \beta\right), 1.15(3 \mathrm{H}, \mathrm{s}, 1-\mathrm{Me} \alpha), 1.20$ (3H, s, 1-Me $\left.\beta\right)$, $1.45(1 \mathrm{H}, \mathrm{t}, J$ 12, 2-H $\beta), 1.50,1.61 .1 .63($ each $1 \mathrm{H}, \mathrm{br} \mathrm{s}, \mathrm{OH} \times 3), 1.67\left(1 \mathrm{H}, \mathrm{dd}, J 7,13.5,2^{\prime}-\mathrm{H}\right), 1.67$ $(3 \mathrm{H}, \mathrm{t}, J 1.5,5 '-\mathrm{Me}), 1.81(1 \mathrm{H}, \mathrm{dd}, J$ 6, 13.5, 2'-H), $1.83(1 \mathrm{H}, \mathrm{ddd}, J 2,3.5,12,2-\mathrm{H \alpha}), 1.92(6 \mathrm{H}, \mathrm{s}$, 5-Me, 9'-Me), 1.95, 1.97 (each 3H, s, 13-Me, 13'-Me), 2.01 (3H, s, 9-Me), 2.07 (1H, ddd, J 1.5, 9.5,

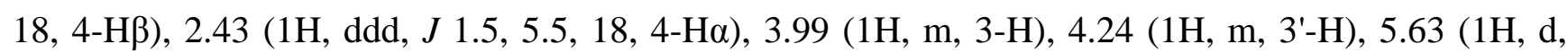
$J$ 15.5, 7'-H), 5.63 (1H, m, 4'-H), 6.22 (1H, br d $\left.J 11.5,10^{\prime}-\mathrm{H}\right), 6.27$ (2H, br d, $J$ 9, 14-H, 14'H), 6.35 $(1 \mathrm{H}, \mathrm{d}, J 14,12-\mathrm{H}), 6.37$ (1H, d, $\left.J 15,12^{\prime}-\mathrm{H}\right), 6.39$ (1H, d, $J$ 15.5, 8'-H), 6.45 (1H, dd-like, $J 1,11.5$, 10-H), 6.51 (1H, dd, $J 11.5,14,11-\mathrm{H}), 6.62\left(1 \mathrm{H}, \mathrm{dd}, J 11.5,15,11^{\prime}-\mathrm{H}\right), 6.64(2 \mathrm{H}, \mathrm{m}, 15-\mathrm{H}, 15 '-\mathrm{H})$; ${ }^{13} \mathrm{C}-\mathrm{NMR}(125 \mathrm{MHz}) \delta$ 12.73, 12.81 (13-M, 13'-Me), 13.13 (9'-Me), 18.04 (9-Me), 19.22 (5'-Me), 22.47 (5-Me), 24.37 (1'-Me), 24.40 (1'-Me), 28.76 (1-Me), 30.50 (1-Me), 36.61 (C1), 38.16 (C1'), 41.46 (C4), 42.47 (C2'), 46.68 (C2), 64.86 (C3), 65.28 (C3'), 77.87 (C6'), 89.00 (C7), 98.62 (C8), 118.96 (C9), 124.16 (C11), 124.22 (C6), 124.84 (C11'), 127.22 (C4'), 129.67 (C7'), 130.08, 130.42 (C15, C15'), 131.98 (C10'), 132.74, 133.44 (C14, C14'), 134.53 (C9'), 134.60 (C8'), 135.17 (C10), 136.22, 136.63 (C13, C13'), 137.26 (C5), 137.98, 138.05 (C12, C12'), 139.01 (C5'); HRMS (ESI) m/z calcd for $\mathrm{C}_{40} \mathrm{H}_{54} \mathrm{O}_{3} \mathrm{Na}(\mathrm{M}+\mathrm{Na})^{+}$605.3965, found 605.3962 . 
Ethyl $\quad(2 E, 4 E)-5-[(1 R, 4 R)-1-H y d r o x y-2,6,6-t r i m e t h y l-4-t r i e t h y l s i l y l o x y c y c l o h e x-2-e n-1-y l]-3-$ methylpenta-2,4-dienoate (2b). To a stirred solution of anti-diol 8 [4] (465 $\mathrm{mg}, 1.58 \mathrm{mmol}$ ), $\mathrm{Et}_{3} \mathrm{~N}(0.66 \mathrm{~mL}, 5.4 \mathrm{mmol})$ and $\mathrm{N}, \mathrm{N}$-dimethyl-4-aminopyridine $(19 \mathrm{mg}, 0.16 \mathrm{mmol})$ in dry $\mathrm{CH}_{2} \mathrm{Cl}_{2}$ $(7 \mathrm{~mL})$ was added TESCl $(0.40 \mathrm{~mL}, 2.4 \mathrm{mmol})$ at $0{ }^{\circ} \mathrm{C}$. The mixture was stirred at $0{ }^{\circ} \mathrm{C}$ for $30 \mathrm{~min}$, poured into saturated aq. $\mathrm{NH}_{4} \mathrm{Cl}$ and extracted AcOEt. The extracts were washed with brine, dried and evaporated to give a residue, which was purified by flash CC (AcOEt- hexane, 1:4) to afford TES ether 2b (645 mg, quant.) as a colorless oil: $[\alpha]_{\mathrm{D}}{ }^{26} 157.89$ (c 0.91, MeOH); IR v 3605, $3473(\mathrm{OH}), 1704$ (conj. CO), 1632, $1612(\mathrm{C}=\mathrm{C}) ;{ }^{1} \mathrm{H}-\mathrm{NMR}(300 \mathrm{MHz}) \delta 0.62\left(6 \mathrm{H}, \mathrm{q}, J\right.$ 8, $\left.\mathrm{SiCH}_{2} \times 3\right), 0.87,1.02$ (each $3 \mathrm{H}, \mathrm{s}$, gem-Me), $0.98\left(9 \mathrm{H}, \mathrm{t}, J\right.$ 8, $\left.\mathrm{SiCH}_{2} \mathrm{Me} \times 3\right), 1.28\left(3 \mathrm{H}, \mathrm{t}, J\right.$ 7.5, $\left.\mathrm{OCH}_{2} \mathrm{Me}\right), 1.60(3 \mathrm{H}, \mathrm{m}, 5-\mathrm{Me})$, $1.66\left(2 \mathrm{H}, \mathrm{d}-\mathrm{like}, J\right.$ 8, 2- $\left.\mathrm{H}_{2}\right), 2.27$ (3H, br s, 9-Me), $4.17\left(2 \mathrm{H}, \mathrm{q}, J 7.5, \mathrm{OCH}_{2}\right), 4.28(1 \mathrm{H}, \mathrm{m}, 3-\mathrm{H}), 5.47$ $\left(1 \mathrm{H}\right.$, br s, 4-H), $5.81(1 \mathrm{H}$, br s, $10-\mathrm{H}), 6.14(1 \mathrm{H}, \mathrm{d}, J 16,7-\mathrm{H}), 6.34(1 \mathrm{H}, \mathrm{d}, J 16,8-\mathrm{H}) ;{ }^{13} \mathrm{C}-\mathrm{NMR}$ $(75 \mathrm{MHz}) \delta 4.82(\mathrm{C} \times 3), 6.87(\mathrm{C} \times 3), 14.11,14.31,17.53,22.66,25.16,39.65,44.53,59.71,65.91$, $79.09,119.38,128.70,132.51,136.94,138.15,151.57,167.14$; HRMS (ESI) $m / z$ calcd for $\mathrm{C}_{23} \mathrm{H}_{40} \mathrm{O}_{4} \mathrm{Si}$ $(\mathrm{MH})^{+}$409.2769, found 409.2764.

In the same procedure as preparation of $\left(3 R, 3^{\prime} S, 6^{\prime} R\right)$-gobiusxanthin $(\mathbf{1 a}),\left(3 R, 3^{\prime} R, 6^{\prime} R\right)$-gobiusxanthin (1b) was prepared from the above $(3 R, 6 R)$-dienoate $\mathbf{2 b}$.

(3R,6R)-Dienal 3b: $[\alpha]_{\mathrm{D}}^{27}-194.8$ (c 0.98, MeOH); IR v 3608, 3477 (OH), 1662 (conj. CO), 1627, 1597, $1580(\mathrm{C}=\mathrm{C}) ;{ }^{1} \mathrm{H}-\mathrm{NMR}(300 \mathrm{MHz}) \delta 0.63\left(6 \mathrm{H}, \mathrm{q}, J\right.$ 8, $\left.\mathrm{SiCH}_{2} \times 3\right), 0.88,1.03($ each $3 \mathrm{H}, \mathrm{s}$, gem-Me), $0.97\left(9 \mathrm{H}, \mathrm{t}, J\right.$ 8, $\left.\mathrm{CH}_{2} \mathrm{Me} \times 3\right), 1.60\left(3 \mathrm{H}, \mathrm{t}, J\right.$ 2, 5-Me), $1.68\left(2 \mathrm{H}, \mathrm{d}-\mathrm{like}, J\right.$ 8, 2- $\left.\mathrm{H}_{2}\right), 2.25(3 \mathrm{H}$, d, J 1, 9-Me), $4.30(1 \mathrm{H}, \mathrm{m}, 3-\mathrm{H}), 5.48(1 \mathrm{H}, \mathrm{m}, 4-\mathrm{H}), 5.98(1 \mathrm{H}, \mathrm{br} \mathrm{d}, J 8,10-\mathrm{H}), 6.31,6.46$ (each 1H, d, $J$ 15.5, 7-H, 8-H), $10.10\left(1 \mathrm{H}, J\right.$ 8, CHO); ${ }^{13} \mathrm{C}-\mathrm{NMR}(75 \mathrm{MHz}) \delta 4.83(\mathrm{C} \times 3), 6.83(\mathrm{C} \times 3), 13.38$, $17.48,22.64,25.18,39.77,44.62,65.85,79.16,128.95,129.65,132.13,136.70,140.21,154.02$, 191.51; HRMS (ESI) $\mathrm{m} / z$ calcd for $\mathrm{C}_{21} \mathrm{H}_{36} \mathrm{O}_{3} \mathrm{NaSi}(\mathrm{M}+\mathrm{Na})^{+}$387.2326, found 387.2319.

(3R,6R)-Hexaenel 5b: UV-VIS $\lambda$ 420; IR v 3604, $3446(\mathrm{OH}), 1660$ (conj. CO), 1611, 1600, 1550 $(\mathrm{C}=\mathrm{C}) ;{ }^{1} \mathrm{H}-\mathrm{NMR}(500 \mathrm{MHz}) \delta 0.92,1.04($ each $3 \mathrm{H}, \mathrm{s}$, gem-Me $), 1.46(1 \mathrm{H}$, br s, 3-OH) $1.53(1 \mathrm{H}$, br d, $J$ 2, 6-OH), 1.58 (1H, dd, $J 10,13.5,2-\mathrm{H}), 1.66$ (3H, t, $J$ 2, 5-Me), 1.79 (1H, ddd, $J 1.5,6.5,13.5,2-\mathrm{H}$ ), $1.88(3 \mathrm{H}, \mathrm{d}, J$ 0.5, 13'-Me), 1.94 (3H, d, $J 0.5,9-\mathrm{Me}), 2.03(3 \mathrm{H}, \mathrm{s}, 13-\mathrm{Me}), 4.30(1 \mathrm{H}, \mathrm{m}, 3-\mathrm{H}), 5.57$ (1H, quint-like, $J 1.5,4-\mathrm{H}), 5.79(1 \mathrm{H}, \mathrm{d}, J 16,7-\mathrm{H}), 6,23(1 \mathrm{H}$, br d, $J 11,10-\mathrm{H}), 6.31(1 \mathrm{H}$, br d, $J 12$, 14-H), 6.32 (1H, d, $J 16,8-\mathrm{H}), 6.38$ (1H, d, $J 15,12-\mathrm{H}), 6.69$ (1H, dd, $J 11.5,14.5,15 '-\mathrm{H}), 6.75(1 \mathrm{H}$, $\mathrm{dd}, J 11$ and 15, 11-H), 6.96 (1H, br d, $J 11.5,14-\mathrm{H}), 7.02(1 \mathrm{H}, \mathrm{dd}, J 12,15,15-\mathrm{H}), 9.45(1 \mathrm{H}, \mathrm{s}$, $\mathrm{CHO}) ;{ }^{13} \mathrm{C}-\mathrm{NMR}(125 \mathrm{MHz}) \delta 9.59$ (13'-Me), 13.03 (13-Me), 13.27 (9-Me), 17.89 (5-Me), 22.45, 25.05 (gem-Me), 39.91 (C1), 44.15 (C2), 65.80 (C3), 79.41 (C6), 127.25, 127.27 (C4, C4'), 127.58 (C15'), 131.23 (C14), 131.75 (C10), 132.15 (C7), 134.18 (C8), 136.39 (C9), 137.03 (C13'), 137.17 (C12), 137.58 (C15), 138.76 (C5), 141.48 (C13), 148.76 (C14'), 194.43 (CHO); HRMS (ESI) m/z calcd for $\mathrm{C}_{25} \mathrm{H}_{34} \mathrm{O}_{3} \mathrm{Na}(\mathrm{M}+\mathrm{Na})^{+} 405.2400$, found 405.2396.

(3R,3'R,6'R)-Gobiusxanthin (1b): UV-VIS $\lambda$ 278, 424, 448, 478; CD $\left(1.08 \times 10^{-4} \mathrm{~mol} / \mathrm{L}, \mathrm{EPA}\right)$ $\lambda(\Delta \varepsilon) 209$ (-2.9), $219(-1.9), 241(-9.5), 259(0), 279$ (+4.0), $294(0), 337(-5.0), 369(-0.6)$; IR $v$ 3606, $3446(\mathrm{OH}), 2172(\mathrm{C} \equiv \mathrm{C}), 1568(\mathrm{C}=\mathrm{C}) ;{ }^{1} \mathrm{H}-\mathrm{NMR}(500 \mathrm{MHz}) \delta 0.92(3 \mathrm{H}, \mathrm{s}, 1$ 1'-Me $\alpha), 1.03(\mathrm{H}, \mathrm{s}$, $\left.1^{\prime}-\mathrm{Me} \beta\right), 1.14(3 \mathrm{H}, \mathrm{s}, 1-\mathrm{Me} \alpha), 1.20$ (3H, s, 1-Meß), 1.45 (1H, t, $J$ 12, 2-H $\left.\beta\right), 1.57$ (1H, dd, $J 10$ and 13,

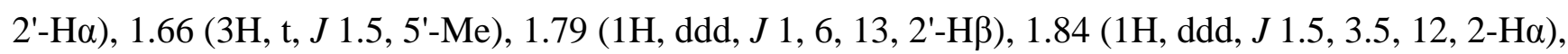


1.92 (6H, s, 5-Me, 9'-Me), 1.95 (3H, s, 13'-Me), 1.97 (3H, s, 13-Me), 2.00 (3H, s, 9-Me), 2.07 (1H, br dd, $J$ 9, 18, 4-H $\beta$ ), 2.43 (1H, ddd, $J 1.5,5.5,18,4-\mathrm{H \alpha}), 3.99(1 \mathrm{H}, \mathrm{m}, 3-\mathrm{H}), 4.29$ (1H, m, 3'-H), 5.57 (1H, m, 4'-H), 5.73 (1H, d, J 15.5, 7'-H), 6.21 (1H, br d, J 11.5, 10'-H), 6.26, 6.27 (each 1H, br d, J 9, 14-H, 14'-H), 6.30 (1H, d, J 15.5, 8'-H), 6.35 (1H, d, J 14.5, 12-H), 6.36 (1H, d, J 15, 12'-H), 6.45 (1H, dd-like, $J 1,11.5,10-\mathrm{H}), 6.51(1 \mathrm{H}, \mathrm{dd}, J 11.5,14.5,11-\mathrm{H}), 6.61\left(1 \mathrm{H}, \mathrm{dd}, J 11.5,15,11^{\prime}-\mathrm{H}\right), 6.64(2 \mathrm{H}$, m, 15-H, 15'-H); ${ }^{13} \mathrm{C}-\mathrm{NMR}$ (125 MHz) $\delta 12.75,12.83$ (13-Me, 13'-Me), 13.20 (9'-Me), 17.95 (5'-Me),

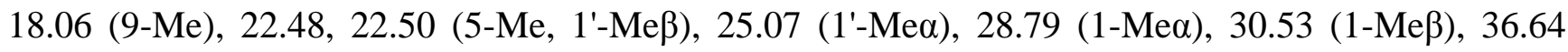
(C1), 39.93 (C1'), 41.51 (C4), 44.19 (C2'), 46.74 (C2), 64.91 (C3), 65.88 (C3'), 79.45 (C6'), 89.04 (C7), 98.66 (C8), 119.00 (C9), 124.19 (C11), 124.29 (C6), 124.86 (C11'), 127.14 (C4'), 130.13, 130.45, (C15, C15') 131.12 (C7'), 132.27 (C10'), 132.81 (C14'), 133.47 (C14), 134.43 (C8'), 134.62 (C9'), 135.20 (C10), 136.25 (C13), 136.67 (C13'), 137.26 (C5), 138.07, 138.09 (C12, C12'), 138.92 (C5'); HRMS (ESI) $m / z$ calcd for $\mathrm{C}_{40} \mathrm{H}_{55} \mathrm{O}_{3}(\mathrm{M}+\mathrm{H})^{+}$583.4146, found 583.4140.

\subsection{Synthesis of Gobiusxanthin Stereoisomers $\mathbf{1 c}-\mathbf{1 f}$}

Ethyl (2E,4E)-5-[(S)-1-Hydroxy-2,6,6-trimethyl-4-oxocyclohex-2-en-1-yl]-3-methylpenta-2,4- dienoate (10). To a mixture of epoxide 9 [9] (2.52 g, $8.57 \mathrm{mmol}), \mathrm{NaHCO}_{3}(800 \mathrm{mg}, 9.5 \mathrm{mmol})$ in $\mathrm{CH}_{2} \mathrm{Cl}_{2}$ $(50 \mathrm{~mL})$ was added DMP $(4.72 \mathrm{~g}, 11.1 \mathrm{mmol})$ in some portions at $\mathrm{rt}$ and the mixture was stirred for a farther $30 \mathrm{~min}$. After $\mathrm{CH}_{2} \mathrm{Cl}_{2}$ was evaporated off, the resulting mixture was diluted with eher-hexane (1:1) and filtered through a pad of Celite. The filtrate was evaporated to afford a residue, which was purified by flash CC (AcOEt-hexane, 2:3) to afford the crude epoxy ketone (2.53 g), which was dissolved in AcOEt (400 mL) and silica gel (70-230 mesh Merck-1.07734; $130 \mathrm{~g})$ was added to it. After being stirred at $\mathrm{rt}$ for $18 \mathrm{~h}$, the mixture was filtered through sintered glass funnel and the filtrate was evaporated. The residue was purified by flash CC (AcOEt-hexane, 35:65) to provide (6S)-enone 10 (2.05 g, 82\% from 9) as colorless solids: Its spectral data were identical with those of previously reported [4] (6R)-enone: [ $\alpha]_{\mathrm{D}}^{22} 340.8$ (c $0.97, \mathrm{MeOH}$ ); HRMS (ESI) $\mathrm{m} / z$ calcd for $\mathrm{C}_{17} \mathrm{H}_{24} \mathrm{O}_{4} \mathrm{Na}$ $(\mathrm{M}+\mathrm{Na})^{+}$315.1567, found 315.1563.

Reduction of enone $\mathbf{1 0}$ with 9-BBN. To a stirred solution of enone $\mathbf{1 0}$ (2.00 g, $6.85 \mathrm{mmol})$ in dry THF $(50 \mathrm{~mL})$ was added dropwise 9-BBN $(0.5 \mathrm{M}$ in THF; $29 \mathrm{~mL} .14 .5 \mathrm{mmol})$ at $0{ }^{\circ} \mathrm{C}$ and the mixture was stirred at $\mathrm{rt}$ for $2 \mathrm{~h}$. The reaction was quenched by addition of $\mathrm{MeOH}(5 \mathrm{~mL})$ followed by 2-aminoethanol $(1 \mathrm{~mL})$ and the mixture was stirred at $\mathrm{rt}$ for $15 \mathrm{~min}$. The mixture was concentrated and the resulting residue was purified by flash $\mathrm{CC}$ (acetone-hexane, 3:7) and then preparative HPLC (COSMOSIL 5SL-II $2 \times 25 \mathrm{~cm}$; MeOH-CH $\left.\mathrm{Cl}_{2}, 2.5: 100\right)$ to afford $(3 R, 6 S)$-syn-diol $11 \mathrm{a}$ (950 mg, $47 \%)$ and $(3 S, 6 S)$-anti-diol $\mathbf{1 1 b}(890 \mathrm{mg}, 44 \%)$ as a colorless viscous oil, respectively. Their spectral data except for optical data were identical with those of previously reported [4] $(3 S, 6 R)$-syn-diol and (3R,6R)-anti-diol 8.

(3R,6S)-Syn-diol 11a: $[\alpha]_{\mathrm{D}}{ }^{21} 140.1$ ( $c$ 0.99 , MeOH); HRMS (ESI) $\mathrm{m} / z$ calcd for $\mathrm{C}_{17} \mathrm{H}_{26} \mathrm{O}_{4} \mathrm{Na}$ $(\mathrm{M}+\mathrm{Na})^{+} 317.1723$, found 317.1714 .

(3S,6S)-Anti-diol 11b: $[\alpha]_{\mathrm{D}}{ }^{20} 240.9$ (c 1.05, MeOH); HRMS (ESI) $\mathrm{m} / z$ calcd for $\mathrm{C}_{17} \mathrm{H}_{26} \mathrm{O}_{4} \mathrm{Na}$ $(\mathrm{M}+\mathrm{Na})^{+} 317.1723$, found 317.1719 . 
In the same procedure as preparation of $\left(3 R, 3^{\prime} S, 6^{\prime} R\right)$-gobiusxanthin (1a), $\left(3 R, 3^{\prime} S, 6^{\prime} S\right)$-gobiusxanthin (1c) and $\left(3 R, 3^{\prime} R, 6^{\prime} S\right)$-gobiusxanthin (1d) were prepared from above $(3 R, 6 S)$-syn-diol 11a and (3S,6S)-anti-diol 11b, respectively. (3S,3'S,6'R)-Gobiusxanthin (1e) and (3S,3'S,6'S)-gobiusxanthin (1f) were prepared by using the enantiomer [11] of $\mathrm{C}_{15}$-phosphonium salt 7. Spectral data except for optical data of compounds $\mathbf{1 c}-\mathbf{f}, \mathbf{1 2 a}, \mathbf{b}, \mathbf{1 3 a}, \mathbf{b}$ and $\mathbf{1 4 a}, \mathbf{b}$ were identical with the corresponding enantiomers $\mathbf{2 a}, \mathbf{b}, \mathbf{3 a}, \mathbf{b}, \mathbf{5 a}, \mathbf{b}$ and diastereomers $\mathbf{1 a}, \mathbf{b}$.

(3R,6S)-Syn-dienoate 12a: $[\alpha]_{\mathrm{D}}{ }^{18} 118.3$ ( $c$ 1.03, MeOH); HRMS (ESI) $\mathrm{m} / z$ calcd for $\mathrm{C}_{23} \mathrm{H}_{40} \mathrm{O}_{4} \mathrm{NaSi}$ $(\mathrm{M}+\mathrm{Na})^{+} 431.2588$, found 431.2589 .

(3R,6S)-Syn-dienal 13a: $[\alpha]_{\mathrm{D}}{ }^{18} 159.4$ ( $c$ 1.04, MeOH); HRMS (ESI) $m / z$ calcd for $\mathrm{C}_{21} \mathrm{H}_{36} \mathrm{O}_{3} \mathrm{NaSi}$ $(\mathrm{M}+\mathrm{Na})^{+}$387.2326, found 387.2321.

(3R,6S)-Syn-apocarotenal 14a: HRMS (ESI) $\mathrm{m} / \mathrm{z}$ calcd for $\mathrm{C}_{25} \mathrm{H}_{34} \mathrm{O}_{3} \mathrm{Na}(\mathrm{M}+\mathrm{Na})^{+} 405.2400$, found 405.2391 .

$\left(3 R, 3^{\prime} R, 6\right.$ 'S)-Gobiusxanthin (1c): CD $\left(1.07 \times 10^{-4} \mathrm{~mol} / \mathrm{L}, \mathrm{EPA}\right) \lambda(\Delta \varepsilon) 205(-2.0), 209(0), 216$ (+1.5), $227(+0.5), 246(+2.1), 277(+3.0), 304(0), 325(-0.2), 345(0)$; HRMS (ESI) $\mathrm{m} / \mathrm{z}$ calcd for $\mathrm{C}_{40} \mathrm{H}_{54} \mathrm{O}_{3} \mathrm{Na}(\mathrm{M}+\mathrm{Na})^{+}$605.3965, found 605.3965.

(3S,6S)-Anti-dienoate 12b: $[\alpha]_{\mathrm{D}}{ }^{19} 164.4$ (c $1.07, \mathrm{MeOH}$ ); HRMS (ESI) $\mathrm{m} / z$ calcd for $\mathrm{C}_{23} \mathrm{H}_{40} \mathrm{O}_{4} \mathrm{NaSi}$ $(\mathrm{M}+\mathrm{Na})^{+} 431.2588$, found 431.2587 .

(3S,6S)-Anti-dienal 13b: $[\alpha]_{\mathrm{D}}{ }^{18} 265.8$ ( $c$ 0.97, MeOH); HRMS (ESI) $m / z$ calcd for $\mathrm{C}_{21} \mathrm{H}_{36} \mathrm{O}_{3} \mathrm{NaSi}$ $(\mathrm{M}+\mathrm{Na})^{+}$387.2326, found 387.2321.

(3S,6S)-Anti-apocarotenal 14b: HRMS (ESI) $\mathrm{m} / z$ calcd for $\mathrm{C}_{25} \mathrm{H}_{34} \mathrm{O}_{3} \mathrm{Na}(\mathrm{M}+\mathrm{Na})^{+} 405.2400$, found 405.2391 .

(3R,3'S,6'S)-Gobiusxanthin (1d): CD $\left(9.91 \times 10^{-5} \mathrm{~mol} / \mathrm{L}, \mathrm{EPA}\right) \lambda(\Delta \varepsilon) 205(-4.5), 219(0), 242$ (+8.0), $259(0), 279(+4.4), 294(0), 338(+4.9), 367(+0.7)$; HRMS (ESI) $m / z$ calcd for $\mathrm{C}_{40} \mathrm{H}_{54} \mathrm{O}_{3} \mathrm{Na}$ $(\mathrm{M}+\mathrm{Na})^{+} 605.3965$, found 605.3956 .

(3S,3'S,6'R)-Gobiusxanthin (1e): CD $\left(9.68 \times 10^{-5} \mathrm{~mol} / \mathrm{L}\right.$, EPA) $\lambda(\Delta \varepsilon) 208(0), 214(-1.4)$, $229(-0.3), 244(-1.2), 276(-2.3), 289(0), 330(+0.3), 352(0)$; HRMS (ESI) $\mathrm{m} / \mathrm{z}$ calcd for $\mathrm{C}_{40} \mathrm{H}_{54} \mathrm{O}_{3} \mathrm{Na}(\mathrm{M}+\mathrm{Na})^{+}$605.3965, found 605.3962 .

(3S,3'S,6'S)-Gobiusxanthin (1f): CD $\left(1.10 \times 10^{-4} \mathrm{~mol} / \mathrm{L}, \mathrm{EPA}\right) \lambda(\Delta \varepsilon) 211(+1.6), 218(+1.1), 241$ (+7.16), $260(0), 279(-3.1), 293(0), 338(+4.2), 369(+0.6)$; HRMS (ESI) m/z calcd for $\mathrm{C}_{40} \mathrm{H}_{53} \mathrm{O}_{3}$ $(\mathrm{M}-\mathrm{H})^{-}$581.3990, found 581.4013.

\subsection{Isolation of Epigobiusxanthin}

The crown-of-thorns starfish Acanthaster planci, collected at the Ootsuki coast, Kochi Prefecture, Japan (10 specimens 1,870 g), was extracted with acetone. The extract was partitioned between ether-hexane (1:1) and water. The organic layer was dried over $\mathrm{Na}_{2} \mathrm{SO}_{4}$ and then evaporated. The residual red-colored oil was chromatographed on silica gel using an increasing percentage of acetone in hexane. The fraction eluted with acetone-hexane (6:4) was subjected to HPLC on silica gel with acetone-hexane (4:6) and then on ODS silica with $\mathrm{CHCl}_{3}-\mathrm{MeCN}_{2}(2: 8)$ to yield epigobiusxanthin $(0.10 \mathrm{mg})$. 


\section{Conclusions}

In summary, we achieved the first total syntheses of gobiusxanthin (1a), 3'-epigobiusxanthin (1b) and other stereoisomers $\mathbf{1 c}-\mathbf{f}$ via the stereoselective Wittig reactions of the $(3 R)$ - $\mathrm{C}_{15}$-acetylenic tri- $n$-butylphosphonium salt $\mathbf{7}$ and its enantiomer with the corresponding $\mathrm{C}_{25}$-apocarotenal stereoisomers $\mathbf{5 a}, \mathbf{b}$ and $\mathbf{1 4 a}, \mathbf{b}$. The absolute configurations of the 3',6'-dihydroxy- $\varepsilon$-end moieties in these two carotenoids were determined by comparison of their CD spectra with those of synthetic samples: 3'S,6'R and 3'S,6'S configurations were deduced for gobiusxanthin and epigobiusxanthin, respectively. In addition, a HPLC separation method for $(3 R)$ - and $(3 S)$-stereoisomers was established by using a chiral column. The HPLC analysis has proved that the stereochemistry of the natural epigobiusxanthin is $3 R, 3^{\prime} S, 6^{\prime} \mathrm{S}$ : natural epigobiusxanthin is $6^{\prime}$-epi-isomer rather than $3^{\prime}$-epi-isomer of gobiusxanthin.

The present research has indicated that HPLC analysis can be a strong tool to determine the absolute stereochemistries of chiral compounds, especially those having multiple chirogenic centers. To do this, in a concerted manner, development of a total synthetic method is essential to supply a sterically-defined authentic sample.

\section{Author Contributions}

Basic idea of the research was proposed by Yumiko Yamano, Takashi Maoka, and Akimori Wda, collaboratively. The synthetic and analytical experiments were designed and performed by Yumiko Yamano, Kotaro Ematsu, and Hiromasa Kurimoto. The isolation of epigobiusxanthin was carried out by Takashi Maoka.

\section{Conflicts of Interest}

The authors declare no conflict of interest.

\section{References}

1. Tsushima, M.; Mune, E.; Maoka, T. Isolation of stereoisomeric epoxy carotenoids and new acetylenic carotenoid from the common freshwater goby Rhinogobius brunneus. J. Nat. Prod. 2000, 63, 960-964.

2. Matsuno, T.; Tsushima, M.; Maoka, T. Salmoxanthin, deepoxysalmoxanthin, and 7,8-didehydrodeepoxysalmoxanthin from the salmon Oncorhynchus keta. J. Nat. Prod. 2001, 64, 507-510.

3. Buchecker, R.; Noack, K. Circular dichroism. In Carotenoids; Britton, G., Liaaen-Jensen, S., Pfander, H., Eds.; Birkhäuser: Basel, Switzerland, 1995; Volume 1B, pp. 63-116.

4. Yamano, Y.; Eno, K.; Hikita, Y.; Kurimoto, H.; Wada, A. Stereocontrolled first total syntheses of salmoxanthin and deepoxysalmoxanthin. Curr. Org. Synth. 2014, in press.

5. Maoka, T.; Akimoto, N.; Terada, Y.; Komemushi, S.; Harada, R.; Saneshima, N.; Sakagami, Y. J. Nat. Prod. 2010, 73, 675-678.

6. Yamano, Y.; Chary, V.M.; Wada, A. Stereoselective total synthesis of the acetylenic carotenoids alloxanthin and triophaxanthin. Org. Biomol. Chem. 2012, 10, 4103-4108. 
7. Yamano, Y.; Yoshizawa, M.; Ito, M. Isolation of $9 Z \beta$-carotene from Dunaliella bardawil and its stereoselective synthesis. J. Nutr. Sci. Vitaminol. 1999, 45, 49-62.

8. Fischli, A.; Mayer, H.; Simon, W.; Stoller, H.J. A synthesis of vitamin A according to the sulfone method. Helv. Chim. Acta 1976, 59, 397-405.

9. Yamano, Y.; Ito, M.; Wada, A. Total synthesis of cucurbitaxanthin A, cycloviolaxanthin and capsanthin 3,6-epoxide by applying a regioselective ring opening of tetrasubstituted epoxides. Org. Biomol. Chem. 2008, 6, 3421-3427.

10. Buschor, D.J.; Eugster, C.H. Synthesis of $\left(3 S, 4 R, 3^{\prime} S, 4^{\prime} R\right)$ - and $\left(3 S, 4 S, 3^{\prime} S, 4^{\prime} S\right)$-crustaxanthin and further compounds with 3,4-dihydroxy $\beta$-end-groups. Helv. Chim. Acta 1990, 73, 1002-1021.

11. Yamano, Y.; Maoka, T.; Wada, A. Synthesis of (3S,3'S)- and meso-stereoisomers of alloxanthin and determination of absolute configuration of alloxathin isolated aquatic animals. Mar. Drugs 2014, 12, 2623-2632.

(C) 2014 by the authors; licensee MDPI, Basel, Switzerland. This article is an open access article distributed under the terms and conditions of the Creative Commons Attribution license (http://creativecommons.org/licenses/by/4.0/). 